\title{
Photoluminescence Imaging Induced by Laser Line Scan: Study for Outdoor Field Inspections
}

Benatto, Gisele Alves dos Reis; Chi, Mingjun; Jensen, Ole Bjarlin; Santamaria Lancia, Adrian Alejo; Riedel, Nicholas; landolo, Beniamino; Davidsen, Rasmus Schmidt; Hansen, Ole; Thorsteinsson, Sune; Poulsen, Peter Behrensdorff

Published in:

Proceedings of 7th World Conference on Photovoltaic Energy Conversion

Link to article, DOI:

10.1109/PVSC.2018.8547416

Publication date:

2018

Document Version

Peer reviewed version

Link back to DTU Orbit

Citation (APA):

Benatto, G. A. D. R., Chi, M., Jensen, O. B., Santamaria Lancia, A. A., Riedel, N., landolo, B., Davidsen, R. S., Hansen, O., Thorsteinsson, S., \& Poulsen, P. B. (2018). Photoluminescence Imaging Induced by Laser Line Scan: Study for Outdoor Field Inspections. In Proceedings of 7th World Conference on Photovoltaic Energy Conversion (pp. 0395-0399). IEEE. https://doi.org/10.1109/PVSC.2018.8547416

\section{General rights}

Copyright and moral rights for the publications made accessible in the public portal are retained by the authors and/or other copyright owners and it is a condition of accessing publications that users recognise and abide by the legal requirements associated with these rights.

- Users may download and print one copy of any publication from the public portal for the purpose of private study or research.

- You may not further distribute the material or use it for any profit-making activity or commercial gain

- You may freely distribute the URL identifying the publication in the public portal 


\title{
Photoluminescence Imaging Induced by Laser Line Scan: Study for Outdoor Field Inspections
}

\author{
Gisele A. dos Reis Benatto ${ }^{1}$, Mingjin Chi ${ }^{1}$, Ole B. Jensen ${ }^{1}$, Adrian A. Santamaria Lancia ${ }^{1}$, Nicholas Riedel ${ }^{1}$, \\ Beniamino Iandolo², Rasmus S. Davidsen², Ole Hansen², Sune Thorsteinsson ${ }^{1}$, Peter B. Poulsen ${ }^{1}$ \\ ${ }^{1}$ Department of Photonics Engineering, Technical University of Denmark, Frederiksborgvej 399, 4000, \\ Roskilde, Denmark \\ ${ }^{2}$ Department of Micro- and Nanotechnology, Technical University of Denmark, Ørsteds Pl., 2800 Kongens \\ Lyngby, Denmark
}

\begin{abstract}
The results of photoluminescence (PL) imaging performed with a laser line scan in a prototype setup are presented in this work. Here we show the PL images with the photovoltaic cell positioned at different distances from the laser line source, therefore with the beam at different intensities and line characteristics. This study is motivated by the time demanding process of acquiring luminescence images for accurate PV inspections in the field, usually made with electroluminescence imaging. The PL imaging induced by a laser line has the potential to be compact enough to be portable and carried out by drones for field inspections.

Index Terms - Photoluminescence imaging, defect detection, PV inspections.
\end{abstract}

\section{INTRODUCTION}

It is essential for owners of both small and large-scale PV installations to have the expected energy generated out of their investment. To ensure that, regular fault detection is highly desirable, however level of detail in inspections is often limited by time, cost and available manpower. Luminescence imaging is a very powerful technique to overcome the time and manpower limitations to accurately detect a large range of major and minor faults in PV modules. In contrast with current field inspection methods, such as infrared thermography and IV tracing, the range of faults that can be detected by luminescence include cell cracks, broken interconnections and potential induced degradation (PID) [1][3].

Luminescence can be induced by electricity, thus called electroluminescence (EL), requiring electrical contacts, which usually is not trivial and can take as much or longer time than the image acquisition of the corresponding PV installation itself. Alternatively, luminescence can also be driven by light therefore called photoluminescence. The sun would be the straightforward way to induce PL; however, as the sunlight is orders of magnitude more intense than the luminescence signal, the need for modulation between open and short circuit to obtain the PL image arises, which again requires electrical connections. Strategies such as contactless EL presented by Johnston et al. [4] and the recent study presented by Bhoopathy et al. [5] using an LED illuminated control cell per bypass diode in the module, are valuable contributions to avoid electrical contact to acquire luminescence images.
However, these strategies present also speed limitations and require tight proximity to the surface of the module.

Additionally, a line scan provides the possibility to detect faults not shown in uniformly illuminated PL, as it is the case with the PL with sunlight excitation. A line scan drives a current throughout the cell, revealing contact related faults and not only structural wafer defects as it does for uniformly illuminated PL [4], [6].

As outdoor EL is more frequently implemented in the field, with several developments for automated inspection [2], [7], an increasing concern rises regarding the limitation that the electrical connections bring to the inspection speed. In the effort to avoid both electrical contact and time restrictions for PV inspections, the development of a line shaped laser light source for inducing PL on PV cells, and modules in the future, is in progress. While here we present the study performed with a prototype setup on a PV cell, the final goal is to be able to induce PL from a single laser light source in a line shape over a PV module, pulsed in synchronization to the camera acquisition for ambient light subtraction. For this goal many parameters have to be optimized such as: beam properties and PL signal correlation at different distances and scanning speeds; effects of the camera moving with laser line beam; proper image reconstruction and image processing for signal improvement and noise removal.

In this study, we address some of these parameters and compare different defects detection in EL and PL images acquired with the laser line positioned at different distances from the sample, which lead to different beam intensities and line characteristics due to the optics construction. The qualitative results of this study will be taken into consideration for the construction of the final prototype in the near future.

\section{EXPERIMENTAL DETAILS AND METHODOLOGY}

\section{A. Samples description}

Three 156 mm x 156 mm PV cells were used in this work: i) a regular mono-crystalline silicon cell (Mono-Si), presenting finger interruptions; ii) a regular multi-crystalline silicon cell (Multi-Si), presenting faults mainly related to electrical contacts; and iii) a multi-crystalline black silicon (Black-Si) textured using reactive ion etch (RIE), presenting electrical 
contact and structural faults related with low minority carrier effective lifetime.

\section{B. Setup and laser line properties}

The luminescence emission peak for silicon-based solar cells at ambient temperature is centered at $1150 \mathrm{~nm}$ [8]. Two camera detectors are commonly used to acquire such emission: i) cooled Si charge-coupled devices (CCD) ii) and short-wave infrared (SWIR) InGaAs sensors. Only the second allows short enough integration times for non-stationary luminescence image acquisition [9] or a fast line scanning imaging, which is crucial for this work. For this reason, an InGaAs camera from Raptor Photonics is used here, together with a long-pass filter to avoid the detection of the laser line.

PL images under low intensity natural light were acquired using an $800 \mathrm{~nm}$ laser diode, with the PV samples in open circuit conditions (Fig. 1). The camera was kept fixed in its position at approximately one meter from the sample. The laser line beam coming from a fiber was shaped into a line with $<5 \%$ of optical power loss and placed at three different distances (indicated in Fig. 1) from the sample: 48 (Distance 1), 80 (Distance 2) and $104 \mathrm{~cm}$ (Distance 3). The distances

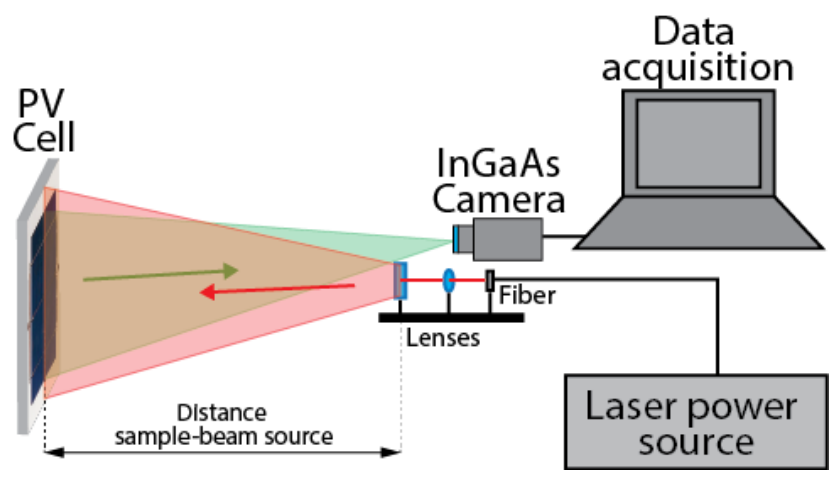

Fig. 1. PL laser line scan setup.

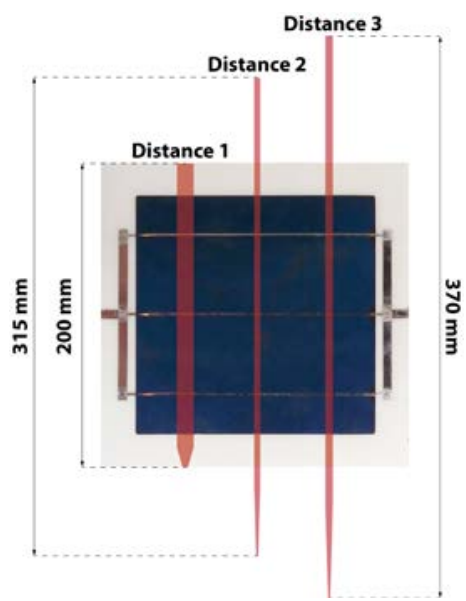

Fig. 2. Laser beam characteristics in relation to a PV sample for distances 1, 2 and 3 from beam source to the sample (see Fig. 1 and text for details). were chosen considering the following criteria: minimum distance to generate a beam wide enough to cover the entire sample; best focus distance for the current optics; and largest distance before the occurrence of optical power loss due to the current optics.

The setup was built as a freely movable setup, as the goal is to have a line scan not attached to an installation structure but from a movable source, ideally a drone. The line beam was manually scanned over the cell area and performed in approximately $4 \mathrm{~s}$, while the images were acquired under 28 ms exposure time. Every PL line image from the scan was stacked together using the software ImageJ and the standard deviation of the stack was used as the final spatially resolved PL image. The beam sizes and characteristics at different distances are illustrated in Fig. 2. The thickness of the each beam was 11, 4 and $5 \mathrm{~mm}$ respectively.

Table 1 shows the approximate laser beam line light intensity in terms of sun irradiance (corresponding to $1000 \mathrm{~W}$ $\mathrm{m}^{-2}$ ) for the three different distances illustrated in Fig. 2. The values are based on the optical power verified at the beam using a power meter, and calculated using the nominal optical loss of lenses and beam line characteristics. The laser power intensity from the fiber was kept constant (unless indicated in section III.A) for the different distances and samples, however the Black-Si sample presented a relatively lower PL signal than the Mono and Multi-Si sample. Therefore, a higher laser power intensity was applied in order to make qualitative comparison of the images easier.

TABLE I

APPROXIMATE LIGHT INTENSITY OF PL EXCITATION LASER LINE AT DIFFERENT DISTANCES FROM SAMPLE TO BEAM SOURCE

\begin{tabular}{cccc}
\cline { 2 - 4 } & \multicolumn{3}{c}{ Laser line intensity in Suns } \\
\cline { 2 - 4 } & Distance 1 & Distance 2 & Distance 3 \\
\hline Mono-Si and Multi-Si & 4.8 & 8.4 & 5.8 \\
\hline Black-Si & 5.6 & 9.8 & 6.7 \\
\hline
\end{tabular}

\section{RESULTS AND DISCUSSION}

\section{A. Laser intensity and repeated scans comparison}

Fig. 3 shows the Mono-Si sample PL images taken at Distance 2 under different laser line intensities. The manual movement was tentatively performed with the same duration (4 s) and at constant speed for all the scans. However, the scanning total durations differ from each other and the speed during the scans slightly varied. The real time duration of the cell scan calculated from the scanning image sequence in seconds is indicated under each PL image in Fig. 3. Slight variations in speed occurred during the scan, even when the total time was identical, evidenced by longitudinally darker and lighter areas in the cell from top to bottom.

The PL images taken with 5.5 and 7.0 Suns equivalent light intensity presented good PL qualitative results, however, 8.4 Suns showed to be the least sensitive to scanning speed variations than the first two intensities. At the same time, 9.8 


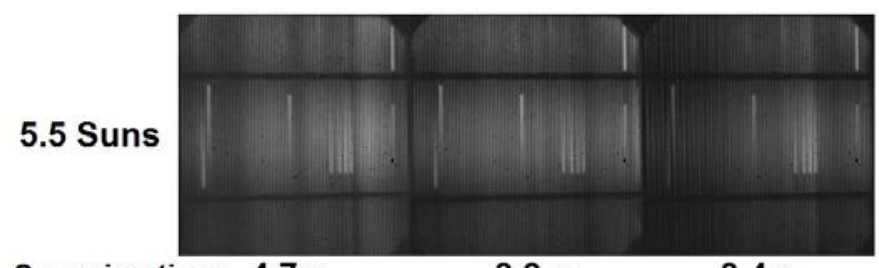

Scanning time: $4.7 \mathrm{~s}$

$3.9 \mathrm{~s}$

$3.4 \mathrm{~s}$
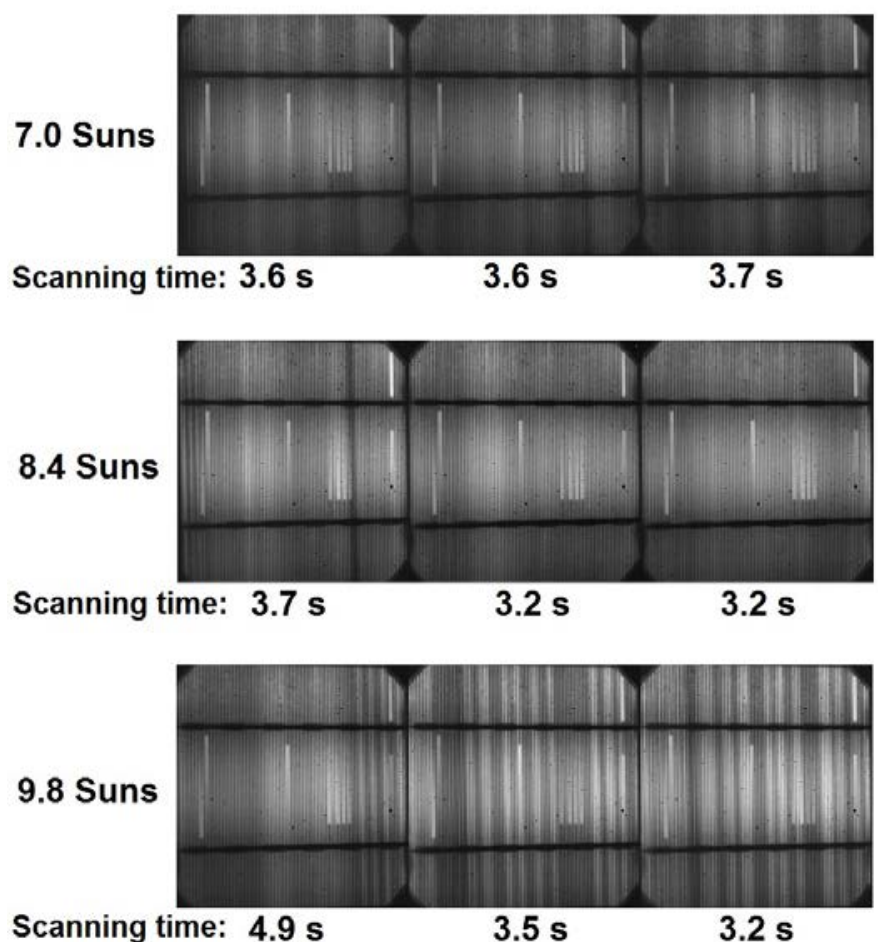

Fig. 3. PL images induced with laser line scan from the Mono-Si at Distance 2, under different laser intensities (in Suns) in three repeated scans with slightly different scanning times (in seconds).

Suns was the light intensity that showed the highest sensitivity to the scanning speed.

From this, we observed that a range of intensities can be selected to acquire good qualitative results. At the same time, while maximum and minimum scanning speeds still need to be evaluated depending on laser intensity and camera exposure time, the results pointed out a high sensitivity of the setup to slight changes in scanning speeds.

\section{B. Distances comparison and fault detection}

The PL images obtained for the three samples using the PL laser line scan are shown in Fig. 4 in comparison with their corresponding EL images (on the right). For all samples, there is a good agreement between EL and PL images. In general, Distance 1 presented a lower PL signal (reflected by the lower light intensity); Distance 2 presented the best defect contrast, thanks to the well-focused beam; and Distance 3 presented the noisiest signal of the three. Even though the images presented distinguable faults in the situations studied, lower PL signal and high levels of noise can be detrimental to the ability to detect some defects, therefore the focus of the beam will be taken specially into consideration in the future setup productions and applications.

At all distances the PL final image had some dependency to the speed homogeneity of the scan. The PL images are markedly more intense in the areas that the beam passed slower, and they are darker where it was faster. The images can also have more separation between one line and the other during image reconstruction when the beam passed slower. The line shape is characterized by a lower intensity towards the bottom of the beam (see Fig. 2). This creates the darker area in the bottom of all PL images from Fig. 4 with reference to the Mono-Si sample EL image.

Mono-Si sample PL shows clearly several finger interruptions, though EL shows few of them that do not appear in PL with this current setup. Finger interruptions are a contact related fault; therefore, its detection is not possible for fully illuminated PL. On the other hand, a line scan implements a driving force for lateral currents (i.e. a contactless EL effect) in the non-illuminated cell regions. This only happens due to the high carrier mobility c-Si PV cells [6].

The Multi-Si sample shows contact related faults from the right side to the center of the cell in the EL image. These areas appear bright in the PL images, indicating a higher series resistance in such areas. This type is an example of fault that could be identified by either luminescence techniques individually.

For the Black-Si sample, comparing PL and EL images was essential to differentiate between electrical and wafer structural defects. The darker region in the central bottom area in the EL image would normally indicate a reduced minority carrier lifetime fault, due to its shape and characteristics. However, when compared with PL, it reveals to be a contact problem, since PL images are directly correlated with the reduced minority carrier lifetime and this fault would appear darker as well in the PL image [10]. Furthermore, this darker region in EL does not allow distinguishing the apparent structural defect with a mesh shape that starts at the top left of the sample and extends until the bottom. The lower minority carrier effective lifetime may be due to higher-than-average surface damage induced by RIE, or by lower-than-average quality of the front surface passivation. A long horizontal micro crack was better identified in the PL images, hidden by the contact fault in the EL image. Firing defects leading to higher series resistance were also revealed in PL images only, however the scanning speed dependency might have enhanced brightness in the firing defect corresponding areas, especially in Distances 1 and 2. Defects originated during firing appear to be common when using Black Si as surface texturing. It is not conclusive, but the contact fault was not directly identifiable in with PL only. The comparison between EL and PL makes the cell diagnosis much more certain in the case of this sample. 


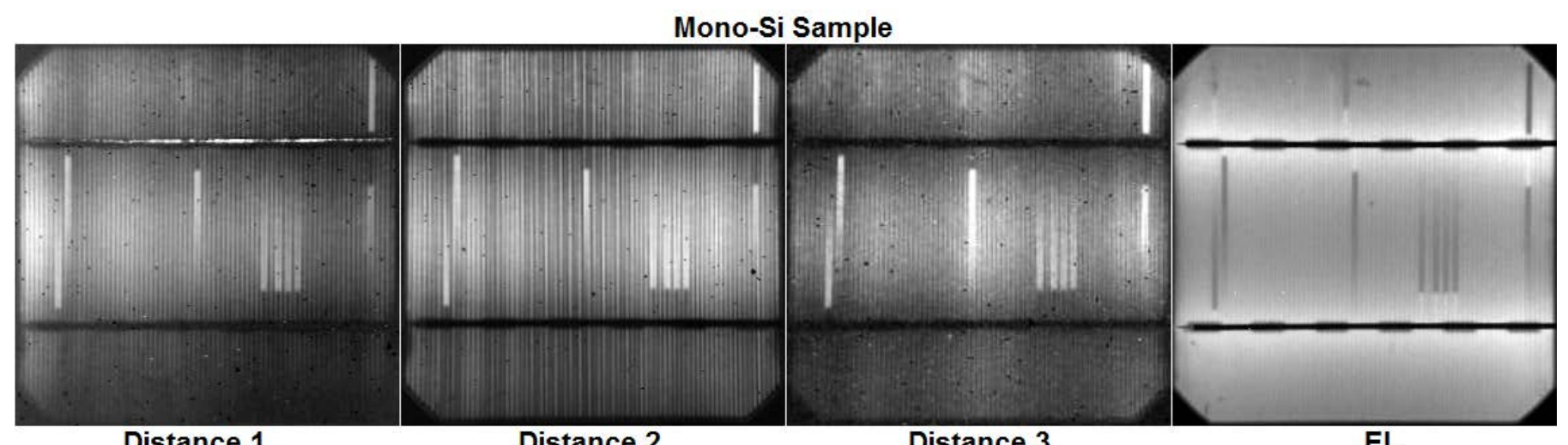

Distance 1

Distance 2

Distance 3

EL

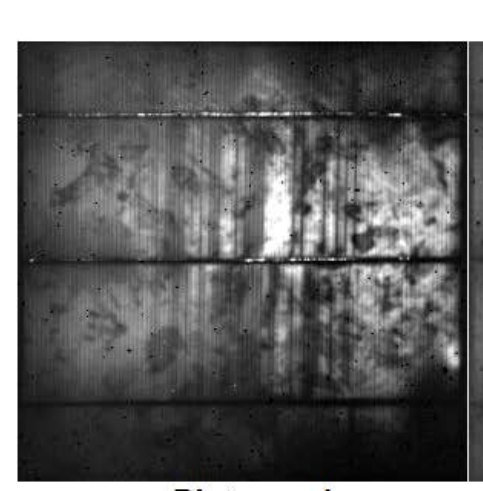

Distance 1

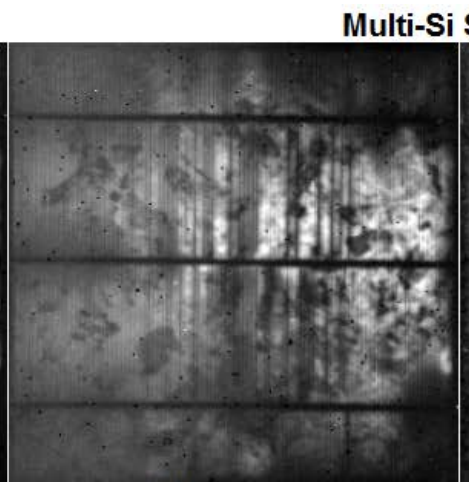

Distance 2

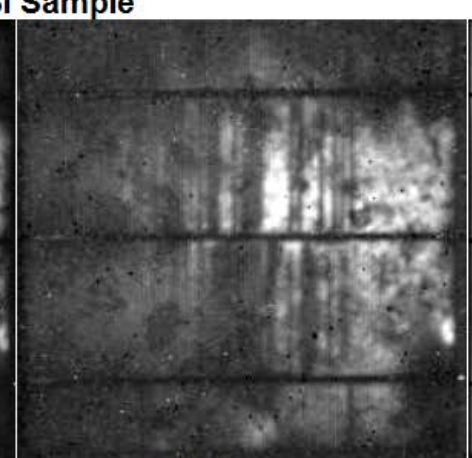

Distance 3

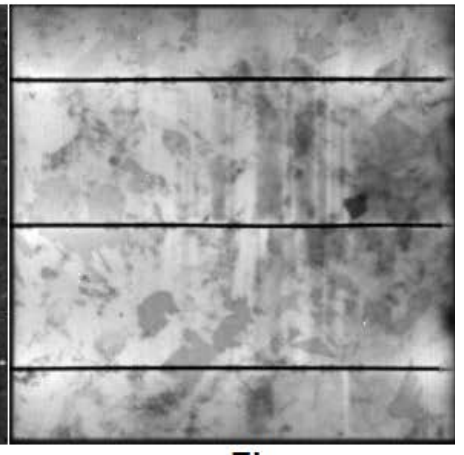

EL

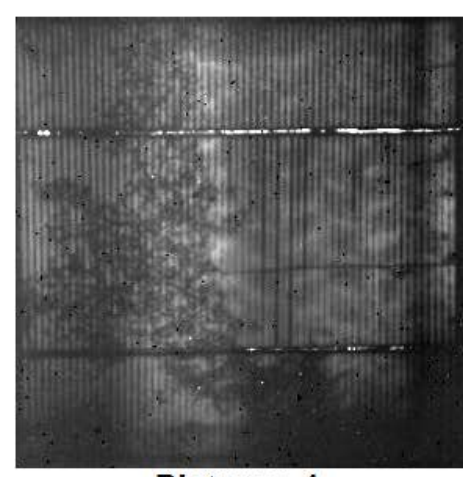

Distance 1

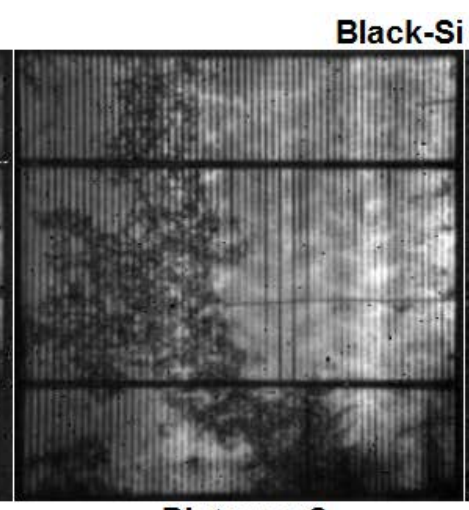

Distance 2

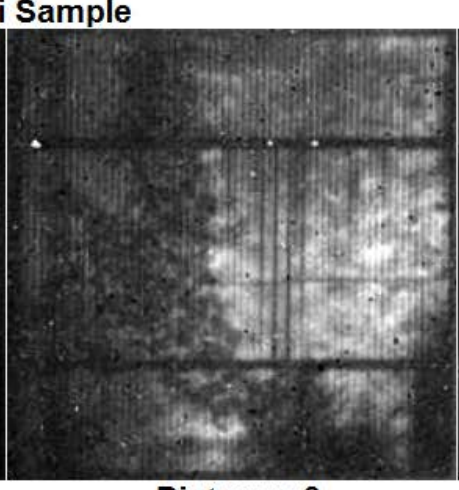

Distance 3

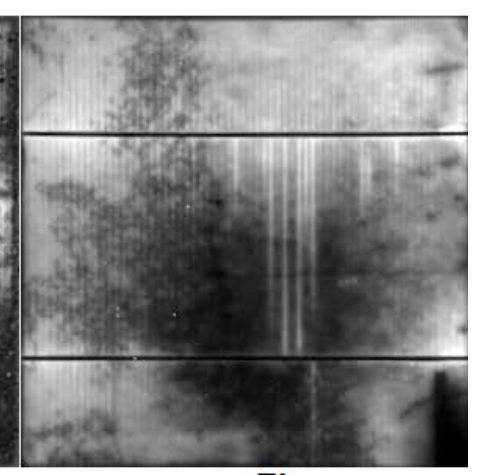

EL

Fig. 4. PL images induced with laser line scan from the Mono-Si, Multi-Si and Black-Si samples at three different distances (see text for details) from the beam source in comparison with the corresponding EL image to the right.

\section{CONCLUSIONS}

In this work we presented the PL images with laser line scan on different samples, laser intensities and beam sizes (corresponding to different distances sample-beam source). The PL images were performed on three different samples and compared with their EL images.

The PL induced by a laser line scan with different laser intensities pointed out a strong sensitivity of the prototype setup to slight variations in the scanning speed (not necessarily scanning duration in the time scale studied), more accentuated in 9.8 Suns laser intensity with the Mono-Si sample. This fact guides the development of precise mechanical scan for the next prototype and a trending limit for the laser line intensity. Moreover, the study of maximum and minimum scanning speeds must be evaluated depending on laser intensity and camera exposure time in a future work.

PL images acquired at different distances indicated that non-well focused laser lines could lead to lower PL signal and 
noise in the images that could be crucial for some defects diagnosis, therefore the focus of the beam and minimal optical power loss at higher distances will be taken especially into consideration for a setup for full module PL line scan.

The PL line scan potential to identify the same or more faults than EL has been observed, but the combination of techniques leaded to a more complete report in the case of the Black-Si sample.

\section{ACKNOWLEDGEMENTS}

The authors acknowledge the financial support from Innovation Fund Denmark for the project 6154-00012B DronEL - Fast and accurate inspection of large photovoltaic plants using aerial drone imaging.

\section{REFERENCES}

[1] M. Köntges et al., "Review of Failures of Photovoltaic Modules,” IEA-Photovoltaic Power Syst. Program., pp. 1-140, 2014.

[2] J. B. S. Koch, T. Weber, C. Sobottka, A. Fladung, P. Clemens, "Outdoor Electroluminescence Imaging of Crystalline Photovoltaic Modules: Comparative Study between Manual Ground-Level Inspections and Drone-Based Aerial Surveys," 32nd Eur. Photovolt. Sol. Energy Conf. Exhib., pp. 1736-1740, 2016.

[3] M. Bliss, X. Wu, K. G. Bedrich, J. W. Bowers, T. R. Betts, and
R. Gottschalg, "Spatially and spectrally resolved electroluminescence measurement system for photovoltaic characterisation,” IET Renew. Power Gener., vol. 9, no. 5, pp. 446-452, 2015.

[4] S. Johnston, "Contactless electroluminescence imaging for cell and module characterization," 2015 IEEE 42nd Photovolt. Spec. Conf. PVSC 2015, 2015.

[5] R. Bhoopathy, O. Kunz, M. Juhl, T. Trupke, and Z. Hameiri, "Outdoor photoluminescence imaging of photovoltaic modules with sunlight excitation,” Prog. Photovoltaics Res. Appl., no. July, pp. 14-16, 2017.

[6] I. Zafirovska, M. K. Juhl, J. W. Weber, J. Wong, and T. Trupke, "Detection of Finger Interruptions in Silicon Solar Cells Using Line Scan Photoluminescence Imaging,” IEEE J. Photovoltaics, pp. 1-7, 2017.

[7] G. A. dos Reis Benatto et al., "Development of outdoor luminescence imaging for drone-based PV array inspection," 44th IEEE Photovolt. Spec. Conf., 2017.

[8] W. S. Yoo, K. Kang, G. Murai, and M. Yoshimoto, "Temperature Dependence of Photoluminescence Spectra from Crystalline Silicon,” ECS J. Solid State Sci. Technol., vol. 4, no. 12, pp. P456-P461, 2015.

[9] J. Adams et al., "Non-Stationary Outdoor EL-Measurements with a Fast and Highly Sensitive InGaAs Camera," 32nd Eur. Photovolt. Sol. Energy Conf. Exhib., 2016.

[10] T. Trupke, R. Bardos, M. Schubert, and W. Warta, "Fast Photoluminescence Imaging of Silicon Wafers," IEEE 4th World Conf. Photovolt. Energy Convers., pp. 928-931, 2006. 